\title{
Spatial entanglement of twin quantum images
}

\author{
Patrick Navez, Enrico Brambilla, Alessandra Gatti, and Luigi A. Lugiato \\ Istituto Nazionale per la Fisica della Materia, Dipartimento di Scienze CC FF MM, Università dell'Insubria, via Valleggio 11, \\ 22100 Como, Italy \\ (Received 11 January 2001; published 14 December 2001)
}

\begin{abstract}
We show that the spatial entanglement of two twin images obtained by parametric down conversion is complete, i.e., concerns both amplitude and phase. By considering a homodyne detection scheme, which allows comparison of field quadrature components of the two images pixel by pixel, Einstein-Podolsky-Rosen correlations are shown to exist between symmetrical pixels of the two images. The best possible correlation is obtained by adjusting the phase profile of the local oscillator in the amplification area. The results for quadrature components hold even in the absence of any input image, i.e., for pure parametric fluorescence. In this case, they are not related to intensity and phase fluctuations.
\end{abstract}

DOI: 10.1103/PhysRevA.65.013813

PACS number(s): 42.50.Dv, 03.67.- a, 42.65. $-\mathrm{k}$

\section{INTRODUCTION}

Optical systems that display quantum entanglement properties in the spatial domain are of great interest for applications, since the amount of information that can be manipulated and processed in parallel exploiting quantum correlation effects increases substantially with respect to the case of single-mode beams. Recently, there has been a rise of interest in the utilization of spatially entangled beams in optical imaging (quantum imaging).

Photons produced by spontaneous down convertion in parametric crystals, in the regime of single-photon pair generation, have been proposed for several imaging applications (see, e.g., [1,2]). In this case, the entanglement concerns the propagation directions (the transverse momentum) of the two photons. However, entanglement exists also at the level of a large number of down-converted photons, where it results in nonclassical spatial correlations between continuous variables of the two down-converted beams. This is the regime that will be investigated in this paper. Among the possible uses of this kind of spatial entanglement, recent proposals concern the possibility of teleporting the quantum state of an optical image [3], and the possibility of measuring small displacements of an optical beam with a sensitivity going beyond the standard quantum limit [4].

We consider the field generated through the process of frequency down conversion in a traveling-wave optical parametric amplifier (OPA). In [5,6], it was demonstrated that such a system, when coupled with an appropriate classical imaging device, is able to generate two symmetrical amplified copies of an injected input image that are strongly correlated one to each other: they indeed display synchronized local intensity fluctuations at the level of quantum noise, and for this reason they may be referred to as twin images.

Here we present new results, that consolidate and complete the picture, showing that the two output images are locally correlated, not only with respect to intensity fluctuations, but also to "phase" fluctuations. We consider a phasesensitive homodyne detection scheme that allows us to compare the fluctuations of field quadrature components from two corresponding pixels of the two output images. We find in general that, for an arbitrary quadrature component char- acterized by a phase $\phi_{L}$, the difference between the fluctuations measured in two symmetrical pixels displays exactly the same spectrum as the sum of the fluctuations in the orthogonal quadrature component $\phi_{L}+\pi / 2$. The common value can be reduced well below the shot noise level over the whole image area, provided that the amplification is large enough and the phase $\phi_{L}$ is correctly adjusted. Optimization of the spatial profile of the phase is crucial to obtain a large level of quantum correlations between symmetrical pixels for the quadrature component $\phi_{L}$ and anticorrelation for the quadrature component $\phi_{L}+\pi / 2$. Thus, the twin images exhibit a complete Einstein-Podolsky-Rosen (EPR) [7] entanglement with respect to continuous variables [8].

Since quantum correlations are shown to exist for any couple of symmetrical pixels over the whole area of the output images, we speak of spatial quantum entanglement. The system exhibits a spatial realization of the EPR paradox for two orthogonal quadrature components of the output field similar to that shown in [9] for the case of the parametric oscillator below threshold. In comparison with the analysis of [9], we consider here also the case in which a coherent image is injected into the system. Furthermore, the consideration of an OPA, instead of an optical parametric oscillator with spherical mirrors, allows us to obtain here completely analytical results.

In the presence of an input image, the mean output field is different from zero, and therefore amplitude (i.e., intensity) and phase fluctuations correspond to special cases of quadrature fluctuations. Therefore, our analysis shows in a rather straightforward way that in symmetrical pixels not only quantum intensity fluctuations are strongly correlated, but phase fluctuations are anticorrelated in the same amount.

The paper is divided as follows. After a presentation of the optical image amplification scheme in Sec. II, in the third section, we study the fluctuation spectrum of the field quadrature components, as it would be measured in the proposed homodyne detection scheme. The fourth section is devoted to the discussion of amplitude and phase fluctuations. The final section includes conclusions and perspectives.

\section{OPTICAL IMAGE AMPLIFICATION SCHEME}

The experimental procedure to generate a pair of quantum entangled images through the process of parametric down 


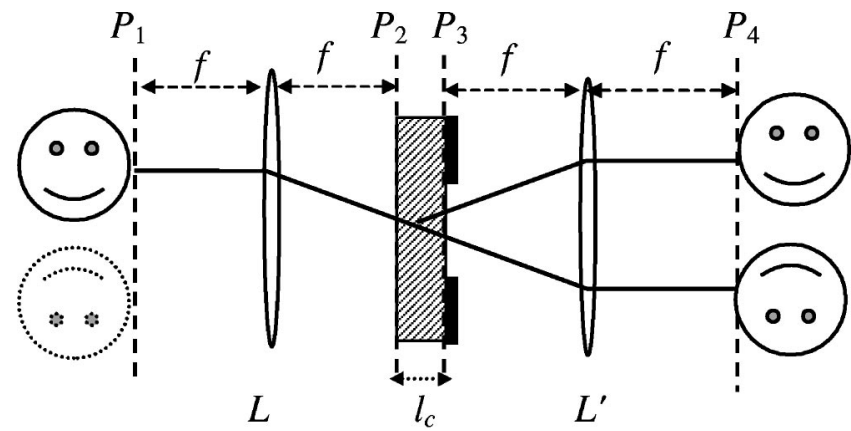

FIG. 1. Schematic diagram of the parametric image amplifier. The two-lens telescopic system enclosing a $\chi^{2}$ crystal produces two amplified copies of the input image that are strongly quantum correlated to each other, thereby the name twin images. The device is phase sensitive when the input image is symmetrical, phase insensitive when it is confined in the upper half of plane $P_{1} . f$ is the focal distance of the lenses.

conversion close to the degenerate frequency has been discussed in previous papers $[5,6]$ and may be summarized as follows. The $\chi^{(2)}$ crystal is enclosed between two lenses $L$ and $L^{\prime}$ of focal length $f$, as shown in Fig. 1. We take the $z$ axis as the main light propagation direction and indicate with $\vec{x}=(x, y)$ the point coordinates in a generic transverse plane. Not shown in the figure is the coherent pump field that activates the process of down conversion and that we take as an ideal classical monochromatic plane wave of frequency $\omega_{p}$, propagating inside the crystal along the $z$ direction.

The crystal slab of width $l_{c}$, ideally infinite in the transverse directions, is cut for type I quasicollinear phase matching at the degenerate frequency $\omega_{p} / 2$. Under these assumptions, each elementary down-conversion process corresponds to the splitting of a pump photon of frequency $\omega_{p}$ into a pair of photons of frequencies $\omega_{p} / 2+\Omega$ and $\omega_{p} / 2-\Omega$, propagating with the same polarization and with opposite transverse wave-vectors $\vec{q}$ and $-\vec{q}$.

Considering only frequencies such that $\Omega \ll \omega_{p}$, the down-converted signal field may be described within the quasimomochromatic approximation by a unique envelope operator $a(z, \vec{x}, t)$. Assuming the validity of the paraxial approximation, which implies that the light beams propagate forming small angles around the mean propagation direction $z, a(z, \vec{x}, t)$ is slowly varying with respect to the carrier wave $\exp \left(i k_{s} z-i \omega_{p} t / 2\right), k_{s}$ being the wave number of the signal at the degenerate frequency. In order to shorten notations, we shall use the definitions $a_{1}(\vec{x}, t)=a(z=-2 f, \vec{x}, t), a_{2}(\vec{x}, t)$ $=a(z=0, \vec{x}, t), \quad a_{3}(\vec{x}, t)=a\left(z=l_{c}, \vec{x}, t\right), \quad a_{4}(\vec{x}, t)=a\left(z=l_{c}\right.$ $+2 f, \vec{x}, t)$, for the envelope operators in the input plane $P_{1}$, the entrance plane $P_{2}$ of the $\chi^{(2)}$ crystal slab, its exit plane $P_{3}$, and the output plane $P_{4}$, respectively (see Fig. 1). We denote by $a_{i}(\vec{x}, \Omega), a_{i}(\vec{q}, \Omega) \quad(i=1, \ldots, 4)$ their Fourier transforms in time and in space time, respectively.

In the linear regime, assuming that pump depletion and losses are negligible, the propagation equations inside the crystal take their simplest form in Fourier space [11]

$$
\begin{aligned}
\frac{d}{d z} a(z, \vec{q}, \Omega)= & -i\left[k_{z}(\vec{q}, \Omega)-k_{s}\right] a(z, \vec{q}, \Omega) \\
& +\sigma e^{-i \Delta_{0} z} a^{\dagger}(z,-\vec{q},-\Omega), \\
\frac{d}{d z} a^{\dagger}(z,-\vec{q},-\Omega)= & i\left[k_{z}(-\vec{q},-\Omega)-k_{s}\right] a^{\dagger}(z,-\vec{q},-\Omega) \\
& +\sigma^{*} e^{i \Delta_{0} z} a(z, \vec{q}, \Omega) .
\end{aligned}
$$

The first term on the right-hand side (r.h.s.) describes linear propagation inside the crystal, $k_{z}(\vec{q}, \Omega)$ being the wavevector component along the $z$ axis of a plane-wave mode of frequency $\omega_{p} / 2+\Omega$ and transverse wave-vector $\vec{q}$. The second term in Eqs. (1) describes the nonlinear interaction between different modes; the coupling constant $\sigma$ is proportional to the pump field amplitude and to the nonlinear susceptibility of the medium. The parameter

$$
\Delta_{0}=2 k_{s}-k_{p}
$$

is the phase mismatch along the $z$ direction between signal and pump waves for collinear propagation.

By solving Eqs. (1), we may write explicitly the inputoutput transformation that relates the field operators in the output face of the crystal (plane $P_{3}$ ) to those in the input face (plane $P_{2}$ ); we obtain the relation (see, e.g., [11])

$$
a_{3}(\vec{q}, \Omega)=u(\vec{q}, \Omega) a_{2}(\vec{q}, \Omega)+v(\vec{q}, \Omega) a_{2}^{\dagger}(-\vec{q},-\Omega) .
$$

The gain coefficients $u(\vec{q}, \Omega)$ and $v(\vec{q}, \Omega)$ are given by the expressions

$$
\begin{aligned}
u(\vec{q}, \Omega)= & \exp \left[i\left(k_{z}(\vec{q}, \Omega)-k_{s}-\frac{\Delta(\vec{q}, \Omega)}{2}\right) l_{c}\right] \\
& \times\left[\cosh \left[\Gamma(\vec{q}, \Omega) l_{c}\right]\right. \\
& \left.+i \frac{\Delta(\vec{q}, \Omega)}{2 \Gamma(\vec{q}, \Omega)} \sinh \left[\Gamma(\vec{q}, \Omega) l_{c}\right]\right] \\
v(\vec{q}, \Omega)= & \exp \left[i \left(k_{z}(\vec{q}, \Omega)-k_{s}\right.\right. \\
& \left.\left.-\frac{\Delta(\vec{q}, \Omega)}{2}\right) l_{c}\right] \frac{\sigma}{\Gamma(\vec{q}, \Omega)} \sinh \left[\Gamma(\vec{q}, \Omega) l_{c}\right],
\end{aligned}
$$

where

$$
\begin{gathered}
\Gamma(\vec{q}, \Omega)=\sqrt{|\sigma|^{2}-\frac{\Delta(\vec{q}, \Omega)^{2}}{4}}, \\
\Delta(\vec{q}, \Omega)=k_{z}(\vec{q}, \Omega)+k_{z}(-\vec{q},-\Omega)-k_{p},
\end{gathered}
$$

are the parametric gain and the phase mismatch for the couple of phase-conjugate modes $(\vec{q}, \Omega)$ and $(-\vec{q},-\Omega)$. 
Assuming for simplicity that the down-converted field is ordinarily polarized, all these functions depend only on the modulus of $\vec{q}$, since propagation is isotropic. For $\Omega=0$, perfect phase matching is achieved when

$$
|\vec{q}|=\sqrt{k_{s}^{2}-\left(k_{p} / 2\right)^{2}} \approx \sqrt{k_{s} \Delta_{0}} .
$$

The gain reaches its maximum around this value of $|\vec{q}|$, with a broad plateau of width $q_{0}=\sqrt{k_{s} / l_{c}}$, the variation scale of $|u|$ and $|v|$ in the spatial frequency domain (see [16] for more details).

The purpose of the two lenses shown in Fig. 1 is to map the Fourier plane $\left(q_{x}, q_{y}\right)$ into the physical plane $(x, y)$. In this manner, if an optical image is injected at the degenerate frequency $\omega_{p} / 2$ in the object plane $P_{1}$, the system amplifies portions of this image rather than a band of its $q$ vectors. Indeed, the presence of the lenses converts input-output relation (3) into a formally identical relation between the realspace field operators in the planes $P_{1}$ and $P_{4}$ :

$$
a_{4}(\vec{x}, \Omega)=\bar{u}(\vec{x}, \Omega) a_{1}(-\vec{x}, \Omega)+\bar{v}(\vec{x}, \Omega) a_{1}^{\dagger}(\vec{x},-\Omega),
$$

where

$$
\bar{u}(\vec{x}, \Omega)=-u\left(\frac{2 \pi \vec{x}}{\lambda f}, \Omega\right), \bar{v}(\vec{x}, \Omega)=v\left(\frac{2 \pi \vec{x}}{\lambda f}, \Omega\right),
$$

and $\lambda$ is the wavelength of the down-converted field at degeneracy.

We must underline that all the results that follow do not depend on the particular form of the gain functions, but rely on the fact that they satisfy the following unitarity conditions:

$$
\begin{gathered}
|u(\vec{q}, \Omega)|^{2}-|v(\vec{q}, \Omega)|^{2}=1, \\
u(\vec{q}, \Omega) v(-\vec{q},-\Omega)=u(-\vec{q},-\Omega) v(\vec{q}, \Omega),
\end{gathered}
$$

which guarantee that the free-field commutation rules are preserved

$$
\begin{gathered}
{\left[a_{i}(\vec{q}, \Omega), a_{i}^{\dagger}\left(\vec{q}^{\prime}, \Omega^{\prime}\right)\right]=\delta\left(\vec{q}-\vec{q}^{\prime}\right) \delta\left(\Omega-\Omega^{\prime}\right),} \\
{\left[a_{i}(\vec{q}, t), a_{i}\left(\vec{q}^{\prime}, t^{\prime}\right)\right]=0, \quad(i=1,2,3,4) .}
\end{gathered}
$$

On the other hand, with respect to other systems that exhibit input/output relations of the same form (e.g., optical parametric oscillators, see, e.g., [12]), the large spatial bandwidth $q_{0}$ of the amplifier makes this traveling-wave scheme a good candidate for high-resolution image amplification.

For the scheme of Fig. 1, the region in the transverse plane that may be efficiently amplified without distortion has a linear size on the order of

$$
x_{0}=\frac{\lambda f}{2 \pi} q_{0},
$$

which represents the width of the plateau of the real-space gain functions (8). Such a region has either the shape of a disc of area $\sim S_{0}=x_{0}^{2}$ centered at the origin, or a ring of width $\sim x_{0}$, depending on the possibility to have collinear $\left(\Delta_{0}=0\right)$ or noncollinear $\left(\Delta_{0}>0\right)$ phasematching at $\Omega=0$, respectively. We assume that the input image is a coherent stationary field of frequency $\omega_{p} / 2$ confined in this region of plane $P_{1}$ (see Fig. 1) so that

$$
\left\langle a_{1}(\vec{x}, \Omega)\right\rangle=\sqrt{2 \pi} \delta(\Omega) \alpha_{i n}(\vec{x}) .
$$

As explained in details in $[6,10]$, whenever the input image is symmetric with respect to the system axis, the device works as a phase-sensitive amplifier (see in this connection also [13]). In this case, the phase of the input image must be selected in order to optimize the gain. Assuming the input image is duplicated before amplification by means of a classical imaging device that produces a symmetrical field ditribution [i.e., $\alpha_{i n}(-\vec{x})=\alpha_{i n}(\vec{x})$ ], the system is able to generate in the output plane two amplified copies that are far better correlated in space time than the originals, meaning by this that they display perfectly (in the ideal case) synchronized local intensity fluctuations. It was also demonstrated [6] that in the limit of high gain, the signal-to-noise ratio as measured from a small portion of the input image before duplication is preserved in the corresponding portions of the two output images: noiseless amplification is therefore achieved for both output channels taken separately (see [14] for an experimental observation of noiseless amplification of images).

In [5], an alternative way to generate a pair of quantum correlated images (also called twin images) was considered; it consists of injecting a single-input image asymetrically, for example, by confining it to the upper half of plane $P_{1}$ as shown in Fig. 1. This configuration does not require a duplication system and presents the further advantage that the gain does not depend on the phase of the input field because the systems works as a phase-insensitive amplifier. However, the fidelity with which information is transferred is worse than in the phase-sensitive case, since the signal-to-noise ratio is deteriorated at least by a factor of two in the amplification process (a feature common to all phase-insensitive optical amplifiers [15]).

Most of the results presented in this paper do not depend on the particular injection scheme, so no assumptions are made about the input intensity distribution $\left|\alpha_{i n}(\vec{x})\right|^{2}$. Imperfect detection can be modeled in the usual way, by coupling the output field operator $a_{4}(\vec{x}, t)$ with an independent operator field $a_{N}(\vec{x}, t)$ which acts on the vacuum state. The contribution $a_{N}$ describes the noise added by losses in the detection process; thus, the effective output field measured by a detector of quantum efficiency $\eta \leqslant 1$ is

$$
a_{D}(\vec{x}, t)=\sqrt{\eta} a_{4}(\vec{x}, t)+\sqrt{1-\eta} a_{N}(\vec{x}, t)
$$

and the corresponding photon flux density is

$$
i(\vec{x}, t)=a_{D}^{\dagger}(\vec{x}, t) a_{D}(\vec{x}, t) .
$$

As shown in Fig. 1, at the exit face of the crystal we insert a pupil of area $S_{p}$, an element that is able to eliminate diver- 
gences arising in the calculation of the field mean intensity and correlation functions when dealing with a system of infinite transverse dimensions $[10,12]$. It also determines the characteristic resolution area of the device in the detection plane, which is $S_{R}=(\lambda f)^{2} / S_{p}$. This finite-size optical element introduces a convolution integral with the pupil response function in the r.h.s. of Eq. (7) and, as a consequence, the points of the input image are spread into diffraction spots of area $S_{R}$ in the output image. However, analytical calculations are performed in the low-diffraction limit, assuming that the diffraction spot size $\sqrt{S_{R}}$ is much smaller than both $x_{0}$ and the variation scale of the input image intensity. Considering a single-pixel detector (labeled by index $j$ ) that intercepts the photons arriving on an area $R_{j}$, which is large in comparison with $S_{R}$, the mean value of the measured photocurrent is then [6]

$$
\begin{aligned}
\left\langle i_{j}(t)\right\rangle= & \int_{R_{j}} d \vec{x}\langle i(\vec{x}, t)\rangle \\
= & \eta \int_{R_{j}} d \vec{x}\left|\vec{u}(\vec{x}, 0) \alpha_{i n}(-\vec{x})+\bar{v}(\vec{x}, 0) \alpha_{i n}^{*}(\vec{x})\right|^{2} \\
& +\frac{\eta}{S_{R}} \int_{R_{j}} d \vec{x} \int_{-\infty}^{\infty} \frac{d \Omega^{\prime}}{2 \pi}\left|\bar{v}\left(\vec{x}, \Omega^{\prime}\right)\right|^{2} .
\end{aligned}
$$

The first integral represents the amplified coherent input field, while the second integral is the contribution coming from spontaneous parametric down conversion. The ratio $S_{0} / S_{R}$ gives an evaluation of the number of details of the input image that can be resolved in the detection plane (e.g., with the pixel array of a charge-coupled device camera). Moreover, quantum correlation effects tends to disappear when $S_{R} \rightarrow S_{0}$, since in this limit, the signal and idler photons of each down-converted pair can no more be resolved separately, because of the large diffraction spread in $q$ space. Making $S_{R}$ as small as possible with respect to $S_{0}$ is therefore a necessary requirement that must be taken into account in experiments. However, this leads to an increase of the spontaneous emission contribution that goes at the expense of the visibility of the amplified input image. This last circumstance imposes a lower limit on the intensity of the input image (see $[5,10,12]$ for more details).

\section{FIELD QUADRATURE CORRELATIONS}

A homodyne detection scheme allows the measurement of a particular quadrature component of the field. It is sketched in Fig. 2 and consists of a beam splitter $M$ that combines the output field with a coherent field of much higher intensity $a_{L}(\vec{x})$, which is usually referred to as the local oscillator field (LO). In the balanced version, a 50/50 beam splitter is used, so that the operators associated to the fields coming from the two output ports of the beam splitter, labeled $b$ and $c$, are

$$
a^{b, c}(\vec{x}, t)=\left[a_{4}(\vec{x}, t) \pm a_{L}(\vec{x})\right] / \sqrt{2},
$$

and the effective fields seen by two identical detectors of quantum efficiency $\eta$ in the two ports $b$ and $c$ are

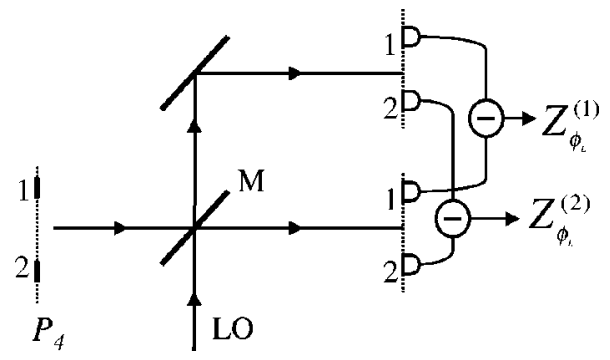

FIG. 2. Homodyne detection scheme for the measurement of the quadrature components of the amplifier output field $Z_{\phi_{L}}^{(1)}$ and $Z_{\phi_{L}}^{(2)}$ from two symmetrical areas of the image plane $P_{4}$ (labeled with 1 and 2). The high-intensity LO field is mixed with the amplifier output field at the symmetric beam splitter $M$.

$$
a_{D}^{b, c}(\vec{x}, t)=\sqrt{\eta} a^{b, c}(\vec{x}, t)+\sqrt{1-\eta} a_{N}^{b, c}(\vec{x}, t),
$$

where $a_{N}^{b, c}(\vec{x}, t)$ describe the noise added in the detection process. When the corresponding intensities are electronically substracted, one obtains a direct measure of the quadrature component of the output field $a_{4}$ selected by the phase of the LO, more precisely

$$
\begin{aligned}
& Z_{\phi_{L}}(\vec{x}, t)=a_{D}^{b^{\dagger \dagger}}(\vec{x}, t) a_{D}^{b}(\vec{x}, t)-a_{D}^{c \dagger}(\vec{x}, t) a_{D}^{c}(\vec{x}, t) \\
& \quad \stackrel{\rightarrow \rightarrow 1}{\rightarrow}{ }_{L}(\vec{x})\left[a_{4}^{\dagger}(\vec{x}, t) e^{i \phi_{L}(\vec{x})}+a_{4}(\vec{x}, t) e^{-i \phi_{L}(\vec{x})}\right],
\end{aligned}
$$

where in the last line we assumed that the LO can be treated as a classical field $\alpha_{L}(\vec{x})=\rho_{L}(\vec{x}) e^{i \phi_{L}(\vec{x})}$. Taking into account the finite size of the pixel detection area $R_{j}$, the measured quantity is

$$
Z_{\phi_{L}}^{(j)}(t)=\int_{R_{j}} d \vec{x} Z_{\phi_{L}}(\vec{x}, t)
$$

We now want to compare the fluctuations of the field quadrature measured in two symmetrical pixels $j=1$ and $j=2$ of the signal and idler image. To this aim, we consider the sum and the difference of the quadrature obtained from two symmetrical detection regions $R_{1}$ and $R_{2}$

$$
Z_{\phi_{L}}^{( \pm)}(t)=Z_{\phi_{L}}^{(1)}(t) \pm Z_{\phi_{L}}^{(2)}(t)
$$

The corresponding fluctuation spectra, defined as

$$
\begin{gathered}
V_{\phi_{L}}^{( \pm)}(\Omega)=\int_{-\infty}^{\infty} d t e^{i \Omega t}\left\langle\delta Z_{\phi_{L}}^{( \pm)}(t) \delta Z_{\phi_{L}}^{( \pm)}(0)\right\rangle, \\
\delta Z_{\phi_{L}}^{( \pm)}(t)=Z_{\phi_{L}}^{( \pm)}(t)-\left\langle Z_{\phi_{L}}^{( \pm)}(t)\right\rangle,
\end{gathered}
$$

describe the degree of correlation between the observables $Z_{\phi_{L}}^{(1)}$ and $Z_{\phi_{L}}^{(2)}$. Using the input-output transformation (7), the commutation rules (10) and the fact that the input image is coherent, we obtain the following relations (the details of the calculation are given in the Appendix):

$$
V_{\phi_{L}}^{(-)}(\Omega)=V_{\phi_{L}^{+}+\pi / 2}^{(+)}(\Omega)
$$




$$
\begin{aligned}
= & (1-\eta)(\mathcal{N})_{L O}+\eta^{2} \int_{R_{1}+R_{2}} d \vec{x} \mid \vec{u}(\vec{x}, \Omega) \alpha_{L}^{*}(\vec{x}) \\
& -\left.\bar{v}^{*}(-\vec{x},-\Omega) \alpha_{L}(-\vec{x})\right|^{2},
\end{aligned}
$$

where

$$
(\mathcal{N})_{L O}=\eta \int_{R_{1}+R_{2}} d \vec{x} \rho_{L}^{2}(\vec{x})
$$

is the shot noise level determined by the LO on the two detectors (we assumed $\left|\alpha_{L}(\vec{x})\right|^{2} \gg\langle i(\vec{x}, t)\rangle$ ). Next, we assume that the LO is symmetric with respect to the system axis, i.e., $\alpha_{L}(\vec{x})=\alpha_{L}(-\vec{x})$. We can write

$$
\begin{aligned}
V_{\phi_{L}}^{(-)}(\Omega)= & V_{\phi_{L}+\pi / 2}^{(+)}(\Omega)=(1-\eta)(\mathcal{N})_{L O} \\
& +\eta^{2} \int_{R_{1}+R_{2}} d \vec{x} F(\vec{x}, \Omega) \rho_{L}^{2}(\vec{x}),
\end{aligned}
$$

where

$$
F(\vec{x}, \Omega)=\left|\bar{u}(\vec{x}, \Omega) e^{-i \phi_{L}(\vec{x})}-\bar{v}^{*}(-\vec{x},-\Omega) e^{i \phi_{L}(\vec{x})}\right|^{2} .
$$

Note that the above expression corresponds to the fluctuation spectrum normalized to shot noise when $\eta=1$ and the pixel side is small with respect to both $x_{0}$ and the scale of variation of $\alpha(\vec{x})$. In this case, $\vec{x}$ in Eq. (25) must be taken as the central point of pixel 1 or pixel 2; due to rotational symmetry around the $z$ axis, the result is the same for both pixels, since $F(\vec{x}, \Omega)=F(-\vec{x}, \Omega)$

A first important result follows from the first equality (23a), according to which $Z_{\phi_{L}}^{(1)}$ and $Z_{\phi_{L}}^{(2)}$ are correlated one to each other exactly to the same extent as the corresponding orthogonal quadrature components $Z_{\phi_{L}+\pi / 2}^{(1)}$ and $Z_{\phi_{L}+\pi / 2}^{(2)}$ are anticorrelated. Second, the common fluctuation spectrum of the two observables $Z_{\phi_{L}}^{(1)}-Z_{\phi_{L}}^{(2)}$ and $Z_{\phi_{L}+\pi / 2}^{(1)}+Z_{\phi_{L}+\pi / 2}^{(2)}$ as given by expression (23b) does not depend on the intensity and phase of the input image (as long as the LO intensity is much larger than that of the output amplified image). Hence, the result is the same in the phase insensitive and in the phase-sensitive scheme, and remains the same even in the absence of an input image at all, i.e., in the case of pure parametric fluorescence. Third, this spectrum may be reduced well below the shot noise level, provided the gain is large enough and the phase of the LO is correctly adjusted. Indeed, assuming that

$$
\phi_{L}(\vec{x})=\frac{1}{2}[\arg \bar{u}(\vec{x}, 0)+\arg \vec{v}(\vec{x}, 0)]=\phi_{\text {opt }}(\vec{x})
$$

over the two detection areas, using the symmetry property of the $\mathrm{LO} \alpha_{L}(-\vec{x})=\alpha_{L}(\vec{x})$ and unitarity relations (9), one obtains from Eq. (26) for $\Omega=0$

$$
F(\vec{x}, \Omega=0)=\frac{1}{[|\vec{u}(\vec{x}, 0)|+|\vec{v}(\vec{x}, 0)|]^{2}},
$$

which goes to zero when $|\vec{u}(\vec{x}, 0)| \sim|\vec{v}(\vec{x}, 0)| \gg 1$. Under conditions of large gain and reasonably large quantum efficiency, almost perfect correlation between the selected quadratures may therefore be obtained.

It is interesting to relate the conditions for obtaining the maximum correlation/anti-correlation with the conditions for obtaining the maximum amplification/deamplification in the phase sensitive configuration. The mean output field is in general

$$
\alpha_{\text {out }}(\vec{x})=\left\langle a_{4}(\vec{x}, t)\right\rangle=\bar{u}(\vec{x}, 0) \alpha_{i n}(-\vec{x})+\bar{v}(\vec{x}, 0) \alpha_{i n}^{*}(\vec{x}),
$$

where we used Eqs. (7) and (12). In the phase-sensitive case where $\alpha_{i n}(-\vec{x})=\alpha_{i n}(\vec{x})$, we may write

$$
\left|\alpha_{\text {out }}(\vec{x})\right|^{2}=G(\vec{x})\left|\alpha_{\text {in }}(\vec{x})\right|^{2}
$$

with the phase-sensitive gain given by

$$
G(\vec{x})=\left|\bar{u}(\vec{x}, 0) e^{i \phi_{i n}(\vec{x})}+\bar{v}(\vec{x}, 0) e^{-i \phi_{i n}(\vec{x})}\right|^{2},
$$

where $\phi_{i n}(\vec{x})$ is the phase distribution of $\alpha_{i n}(\vec{x})$. The maximum value for the gain $G_{\max }(\vec{x})=[|\vec{u}(\vec{x}, 0)|+|\vec{v}(\vec{x}, 0)|]^{2}$ is obtained for

$$
\phi_{i n}(\vec{x})=\phi_{i n}^{\max }(\vec{x})=\frac{1}{2}[\arg \bar{v}(\vec{x}, 0)-\arg \bar{u}(\vec{x}, 0)] .
$$

From Eq. (29) with $\alpha_{i n}(\vec{x})=\left|\alpha_{i n}(\vec{x})\right| \exp \left(i \phi_{i n}^{\max }\right)$ one obtains that in this case, the phase $\phi_{\text {out }}$ of the output field $\alpha_{\text {out }}$ is given by

$$
\phi_{\text {out }}(\vec{x})=\phi_{\text {out }}^{\max }(\vec{x})=\frac{1}{2}[\arg \bar{u}(\vec{x}, 0)+\arg \bar{v}(\vec{x}, 0)]
$$

and therefore coincides with $\phi_{\text {opt }}(\vec{x})$ given by Eq. (27). On the other hand, when the input field phase is selected as $\phi_{i n}(\vec{x})=\phi_{i n}^{\max }(\vec{x})+\pi / 2$, the input field undergoes the maximal deamplification, with the phase-sensitive gain attaining its minimum value $G_{\text {min }}(\vec{x})=[|\vec{u}(\vec{x}, 0)|-|\vec{v}(\vec{x}, 0)|]^{2}$ $=1 / G_{\max }(\vec{x})$. In this case, the output field phase distribution is given by $\phi_{\text {out }}(\vec{x})=\phi_{\text {opt }}(\vec{x})+\pi / 2$. This leads to the following interpretation for the particular form of the LO phase that gives the maximum amount of correlation/anticorrelation between symmetrical pixels: the LO phase that gives the optimum squeezing for $Z_{\phi_{L}}^{(-)}$coincides with the phase of the output field in the phase-sensitive scheme and in conditions of maximal amplification, whereas the optimum squeezing for $Z_{\phi_{L}}^{(+)}$is achieved for the orthogonal phase. Hence, as it will be showed in more detail in the next section, when the input phase is selected for maximal amplification, we may say that amplitude-quadrature fluctuations on symmetric pixels are maximally correlated while phase-quadrature flucta- 
tions are maximally anticorrelated. On the other hand, a LO phase distribution coinciding with that of the output field in conditions of maximal deamplification, permits us to reach the optimal squeezing for $Z_{\phi_{L}}^{(+)}$, while the orthogonal phase gives the best squeezing for $Z_{\phi_{L}}^{(-)}$. In this condition, we can say that phase-quadrature fluctuations on symmetric pixels are correlated, while amplitude-quadrature fluctations are anticorrelated.

Incidentally it should be noted that the above arguments may be easily extended to a phase-sensitive configuration corresponding to the injection of an odd image in the input, that is $\alpha_{i n}(-\vec{x})=-\alpha_{i n}(\vec{x})$. Maximal amplification now takes place when the input phase is orthogonal to the one given by Eq. (32), i.e., $\phi_{i n}(\vec{x})=\phi_{i n}^{\max }(\vec{x})+\pi / 2$. The amplified output field $\alpha_{\text {out }}$ is also odd, but we can have exactly the same situation as for the injection of an even image provided we consider a LO odd with respect to inversion coordinate, and we set $\phi_{\text {opt }} \rightarrow \phi_{\text {opt }}+\pi / 2, \phi_{\text {out }} \rightarrow \phi_{\text {out }}+\pi / 2$.

The results obtained for the observables $Z_{\phi_{L}}^{( \pm)}$closely ressemble the situation of the EPR paradox for continous variables demonstrated in [8], but generalized to many pixels (see also [9]) and to the presence of input images. We notice indeed that the conjugated observables $X_{j}=\int_{-T_{D} / 2}^{T_{D} / 2} d t Z_{\phi_{L}}^{(j)}(t)$ and $P_{j}=\int_{-T_{D} / 2}^{T_{D} / 2} d t Z_{\phi_{L^{+}} / 2}^{(j)}(t)(j=1,2)$ obey the uncertainty rule

$$
\left\langle\delta^{2} X_{j}\right\rangle\left\langle\delta^{2} P_{j}\right\rangle \geqslant \frac{1}{4}\left[T_{D} \int_{R_{1}+R_{2}} d \vec{x} \rho_{L}^{2}(\vec{x})\right]^{2} .
$$

On the other hand, the following combination over the two pixels: $X_{-}=X_{1}-X_{2}$ and $P_{+}=P_{1}+P_{2}$ are commuting observables that can be simultanously determined. When the time of measurement $T_{D}$ is much larger than the inverse of the temporal bandwidth of the OPA, using Eq. (22), the uncertainty of these observables may be directly related to the fluctuations spectrum $V_{\phi_{L}}^{(-)}$

$$
\left\langle\delta^{2} X_{-}\right\rangle=\left\langle\delta^{2} P_{+}\right\rangle=T_{D} V_{\phi_{L}}^{(-)}(\Omega=0) .
$$

For $\eta=1$, an optimal adjustement of the LO phase allows these uncertainties to reach almost a zero value for large amplification and thus to display an apparent violation of the Heisenberg rule

$$
\left\langle\delta^{2} X_{-}\right\rangle\left\langle\delta^{2} P_{+}\right\rangle<\frac{1}{4}\left[T_{D} \int_{R_{1}+R_{2}} d \vec{x} \rho_{L}^{2}(\vec{x})\right]^{2} .
$$

However, it is impractical to synthetyze a LO with the phase variation prescribed by Eq. (27). On the other hand, for a LO with constant phase, the condition (27) concerning the phase of the LO can be exactly satisfied only for a single couple of pixels of area small compared to $S_{0}$, so that the gain functions $|\vec{u}(\vec{x}, 0)|$ and $|\vec{v}(\vec{x}, 0)|$ are nearly uniform over the detection areas. We may however show that by introducing an appropriate curvature, the wave front of the LO field, EPRlike correlations are present for each couple of symmetric

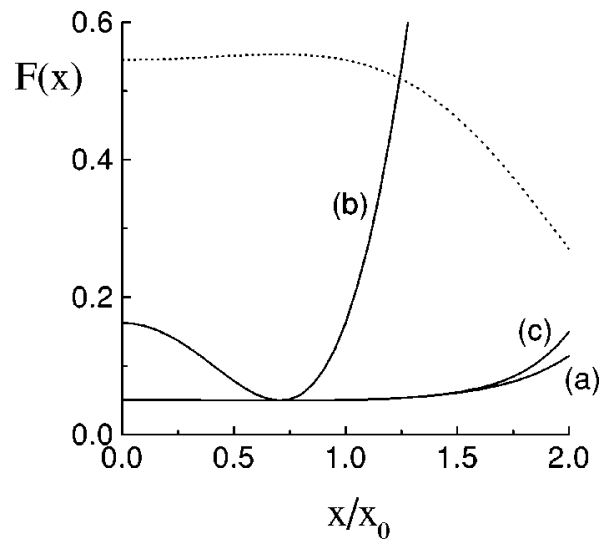

FIG. 3. Plot of the noise reduction factor $F(\vec{x}, 0)$. Subscripts (a) refer to the optimal phase of the LO while (b) and (c) refer, respectively, to a constant phase and to a phase with a quadratic dependence on the distance from the optical axis. The dashed line is the phase-sensitive gain of the OPA [see Eq. (31) divided by a factor 10]. $\Delta_{0} l_{c}=0.5$ and $|\sigma| l_{c}=1.5$.

pixels in the output over the whole gain region $S_{0}$. To this end, we allow the LO phase distribution to have a quadratic dependence on the spatial coordinate (which corresponds to a spherical wave front as one has, e.g., in Gaussian beams). The wave-front curvature is selected in order to have the best fit of the spatial dependance of $\phi_{\text {opt }}(\vec{x})$ in Eq. (27).

Figure 3 plots the function $F(\vec{x}, \Omega=0)$ in the limit where $R_{1}$ and $R_{2}$ are small compared to $S_{0}$ and symmetric. The collinear phase mismatch at degeneracy is $\Delta_{0} l_{c}=0.5$ and the linear gain parameter is $|\sigma| l_{c}=1.5$.

Curve (a) corresponds to the ideal case, with $\phi_{L}$ satisfying condition (27) everywhere in the transverse plane, and leads to a maximal amount of noise reduction in the whole amplification region. In curve (b), the phase of the LO is constant and satisfies condition (27) only in the point of maximum gain $x_{G}$, where perfect phase matching is achieved, i.e., we have $\phi_{L}(\vec{x})=\phi_{\text {opt }}\left(x_{G}\right)$. For our choice of parameters in this point we have $|\bar{u}|^{2} \simeq 5.5$ and $F=(\mid \bar{u}$ $|-| \bar{v} \mid)^{2}=\exp \left(-2|\sigma| l_{c}\right) \simeq 0.05$. Curve (c) is obtained by optimizing the phase with a quadratic term, that is we take $\phi_{L}(\vec{x})=\phi_{\text {opt }}\left(x_{G}\right)+1 / 2\left(d^{2} \phi_{o p t} / d^{2} x\right)\left(x-x_{G}\right)^{2}$; this is the best that can be done with a Gaussian LO and is close to the ideal case.

Notice that in practice, there exist other tricks to perform the phase optimization. For example, one may use a LO with a flat wave front in the detection plane $P_{4}$, and shifts instead the crystal sample away from the focal plane of the lens $L^{\prime}$ by an amount $\delta$. In this case, in fact, the field $a_{4}^{\prime}$ in the detection plane is related to the same field in the absence of the $\delta$ shift by $a_{4}^{\prime}(\vec{x})=a_{4}(\vec{x}) e^{-i k_{s} x^{2} \delta / 2 f^{2}}$. After the interference with a local oscillator, the measured quadrature is $Z_{\phi_{L}}(\vec{x})=a_{4}(\vec{x}) e^{-i\left(\phi_{L}+k_{s} x^{2} \delta / 2 f^{2}\right)}+a_{4}^{\dagger}(\vec{x}) e^{i\left(\phi_{L}+k_{s} x^{2} \delta / 2 f^{2}\right)}, \quad$ so that by properly adjusting the $\delta$ shift, the overall effect is the same as using a LO with a curved wave front. 


\section{PHASE-INTENSITY ENTANGLEMENT OF THE TWIN IMAGES}

Although the phase-sensitive measurement scheme considered in the last section offers a picture of the spatial correlations that can be observed in the output field, intensity correlation measurements are more straightforward to perform experimentally and lead also to interesting effects of quantum noise reduction $[5,6,16]$. The observable that displays reduced fluctuations with respect to the coherent state level is the difference between the direct photocurrents measured from two symmetrical detection region $i_{-}=i_{1}-i_{2}$. The corresponding fluctuation spectrum is

$$
V_{i_{-}}(\Omega)=\int_{-\infty}^{\infty} d t e^{i \Omega t}\left\langle\delta i_{-}(t) \delta i_{-}(0)\right\rangle .
$$

By using Eqs. (7), (10), (13), (14), and (15), and the fact that input image is in a coherent state one obtains after lengthy but elementary calculations (the demonstration is outlined in the Appendix)

$$
\begin{aligned}
V_{i_{-}}(\Omega)= & (1-\eta)\left\langle i_{+}\right\rangle+\eta^{2} \int_{R_{1}+R_{2}} d \vec{x} \mid \bar{u}(\vec{x}, \Omega) \alpha_{\text {out }}^{*}(\vec{x}) \\
& -\left.\bar{v}^{*}(\vec{x},-\Omega) \alpha_{\text {out }}(-\vec{x})\right|^{2}+\eta^{2} \frac{1}{S_{R}} \int_{R_{1}+R_{2}} d \vec{x} \\
& \times \int \frac{d \Omega^{\prime}}{2 \pi}\left[\left|\bar{u}\left(\vec{x}, \Omega^{\prime}\right)\right|^{2}\left|\bar{v}\left(\vec{x}, \Omega+\Omega^{\prime}\right)\right|^{2}-\bar{u}(\vec{x}, \Omega\right. \\
& \left.\left.+\Omega^{\prime}\right) \bar{v}\left(\vec{x},-\Omega-\Omega^{\prime}\right) \bar{u} *\left(\vec{x}, \Omega^{\prime}\right) \bar{v} *\left(\vec{x},-\Omega^{\prime}\right)\right],
\end{aligned}
$$

where $\alpha_{\text {out }}(\vec{x})$ is given by Eq. (29). The shot noise level corresponds to the photocurrent sum $\left\langle i_{+}\right\rangle=\left\langle i_{1}+i_{2}\right\rangle$. The second term on the r.h.s. of Eq. (38) arises from the interference between the amplified input field and the fluorescence field. The last term, which does not depend on the presence of an input field, is a pure noise contribution due to the self interference of the fluorescence field and reduces to zero for $\Omega=0$ because $\bar{v}(\vec{x},-\Omega)=\bar{v}(\vec{x}, \Omega)[5]$.

Using the explicit expression of the amplified input field (29) and the fact that $\bar{u}(\vec{x}, \Omega)=\bar{u}(-\vec{x}, \Omega), \bar{v}(\vec{x}, \Omega)=\bar{v}$ $(-\vec{x}, \Omega)$ and Eq. (9), we find for the zero frequency value of the spectrum

$$
V_{i_{-}}(0)=(1-\eta)\left\langle i_{+}\right\rangle+\eta^{2} \int_{R_{1}+R_{2}} d \vec{x}\left|\alpha_{i n}(\vec{x})\right|^{2} .
$$

As shown in [5], in the case of ideal detection $(\eta=1)$, the noise level of $i_{-}$reduces therefore to the noise of the input image over $R_{1}+R_{2}$. As a consequence, under conditions of large gain, fluctuations are well below the shot noise level.

It is important now to connect with the result for quadrature components obtained in the previous section. To this aim, let us first assume that the input field is strictly different from zero at least in some region of the transverse plane. Second, let us assume that the parametric values are such that the pure noise contribution in $V_{i_{-}}(\Omega)$ [i.e., the last term in Eq. (38)] is negligible and, similarly, that the second term in Eq. (15) may be dropped. Thus, expression (38) reduces to

$$
\begin{aligned}
V_{i_{-}}(\Omega)= & (1-\eta) \mathcal{N}_{-}+\eta^{2} \int_{R_{1}+R_{2}} d \vec{x} \mid \vec{u}(\vec{x}, \Omega) \alpha_{\text {out }}^{*}(\vec{x}) \\
& -\left.\bar{v}^{*}(\vec{x},-\Omega) \alpha_{\text {out }}(-\vec{x})\right|^{2}
\end{aligned}
$$

where

$$
\mathcal{N}_{-}=\eta \int_{R_{1}+R_{2}} d \vec{x}\left|\alpha_{\text {out }}(\vec{x})\right|^{2}
$$

and we used Eq. (29). By comparing with Eqs. (23b) and (24), we see that this expression coincides with $V_{\phi_{L}}^{(-)}(\Omega)$ if we take

$$
\alpha_{L}(\vec{x})=\alpha_{\text {out }}(\vec{x})
$$

This is expected because a LO with the configuration of the output field just picks up the amplitude fluctuations. The relation becomes even more precise in the phase-sensitive case $\alpha_{i n}(\vec{x})=\alpha_{i n}(-\vec{x})$. In this case, assuming $\eta=1$ and that the pixel size is small with respect to both $x_{0}$ and the scale of variation of $\alpha_{\text {out }}(\vec{x})$, one has

$$
\begin{aligned}
\frac{V_{i_{-}}(\Omega)}{\mathcal{N}_{-}}=\widetilde{F}(\vec{x}, \Omega)= & \mid \bar{u}(\vec{x}, \Omega) e^{-i \phi_{\text {out }}(\vec{x})} \\
& -\left.\bar{v}^{*}(\vec{x},-\Omega) e^{i \phi_{\text {out }}(\vec{x})}\right|^{2},
\end{aligned}
$$

where we set $\alpha_{\text {out }}(\vec{x})=\rho_{\text {out }}(\vec{x}) \exp \left(i \phi_{\text {out }}(\vec{x})\right)$, and $\vec{x}$ is the central point of any of the two symmetrical pixels. The result coincides with that of Eq. (25) where $\phi_{L}$ is replaced by $\phi_{\text {out }}$. The link between intensity fluctuations and quadrature fluctuations allows us to analyze the case of phase fluctuations. In the small quantum noise approximation, these concide with the quadrature fluctuations measured with a LO with a phase distribution shifted of $\pi / 2$ with respect to the mean-field phase distribution (that is, the LO that provides the amplitude fluctuations). Therefore, in terms of pixels, we are lead to consider the observables $Z_{\phi_{L}+\pi / 2}^{(j)}(t)$ [see Eq. (20)] with $\phi_{L}=\phi_{\text {out }}$. This naturally induces us to focus on the observable $Z^{(+)}(t)=Z_{\phi_{\text {out }}+\pi / 2}^{(1)}(t)+Z_{\phi_{\text {out }}+\pi / 2}^{(2)}(t)$, see Eq. (21), which measures the degree of anticorrelation between the phase fluctuations in the two symmetrical pixels 1 and 2 . The spectrum $V_{\phi_{L}+\pi / 2}^{(+)}(\Omega)$ coincides with $V_{\phi_{L}}^{(-)}(\Omega)$, which, as we have seen, is identical to $V_{i-}(\Omega)$ given by Eq. (43). Therefore, for large amplification, the fluctuations of $Z^{(+)}(t)$ are well below the shot noise level, which implies that the phase fluctuations in the two symmetrical pixels are strongly anticorrelated, exactly as the amplitude fluctuations are strongly correlated.

One could wonder at this point how is the fluctuation spectrum of the sum of intensities collected from symmetri- 
cal pixels, $i_{+}=i_{1}+i_{2}$. Under the same assumptions that allowed us to write Eq. (43) we find

$$
\begin{aligned}
\frac{V_{i_{+}}(\Omega=0)}{\mathcal{N}_{+}}= & G(\vec{x})+\frac{4|\bar{u}(\vec{x}, 0) \bar{v}(\vec{x}, 0)|^{2}}{G(\vec{x})} \\
& \times \sin ^{2}\left[2 \phi_{i n}(\vec{x})-2 \phi_{i n}^{\max }(\vec{x})\right],
\end{aligned}
$$

where $\mathcal{N}_{+}$is the shot noise level and coincides with $N_{-}$ given by Eq. (41). We notice that both in the case of maximal amplification $\phi_{i n}(\vec{x})=\phi_{i n}^{\max }$, and in the case of maximal deamplification $\phi_{i n}(\vec{x})=\phi_{i n}^{\max }+\pi / 2$, we have that $V_{i_{+}}(\Omega$ $=0) / \mathcal{N}_{+}=G(\vec{x})$. In the latter case, however $G(\vec{x})$ $=G_{\min }(\vec{x})<1$ and the spectrum of fluctuations of the intensity sum is below shot noise. This kind of result was anticipated in the previous section by considering a homodyne measurement performed by means of LO with the same phase distribution of the output field. ${ }^{1}$ However, some caution should be taken when considering a direct measurement of the intensity sum. In fact, at difference from the case of the intensity difference, the spontaneous fluorescence contribution in the noise spectrum of the intensity sum does not vanish at low frequencies. This is a consequence of the fact that spontaneous fluorescence photons are emitted in pairs propagating in symmetrical directions, so that fluctuations in the number of photons collected from symmetrical pixels are strongly correlated; when summing the light intensities from symmetrical pixels, the photon number fluctuations sum coherently instead of canceling one with each other. Moreover, this term could be not negligible compared to the contribution coming from the beating of the mean field with the noise, as the mean field itself results from the deamplification of the input. Indeed, some effect of noise reduction in the intensity sum could be eventually observed only in the presence of a large intensity input, so that the deamplified output intensity is still much larger than the spontaneous fluorescence mean intensity.

\section{CONCLUSION}

In this article, we analyzed extensively a system formed by an optical parametric amplifier with some imaging lenses. Amplification of optical images by OPA has been already studied in the literature [17], but only from a classical viewpoint.

Our results hold both for a phase-sensitive configuration (symmetrical input image) and for a phase insensitive one (asymmetrical injection).

We demonstrated that the two output twin images exhibit a complete spatial EPR entanglement. This was shown, first of all, by considering a pair of orthogonal quadrature components of the output field. In the case of local oscillator symmetrical with respect to the system axis, we found a

\footnotetext{
${ }^{1}$ As it should be by now clear, we hence have that the spectrum of fluctuations of the phase difference is in correspondence below shot noise.
}

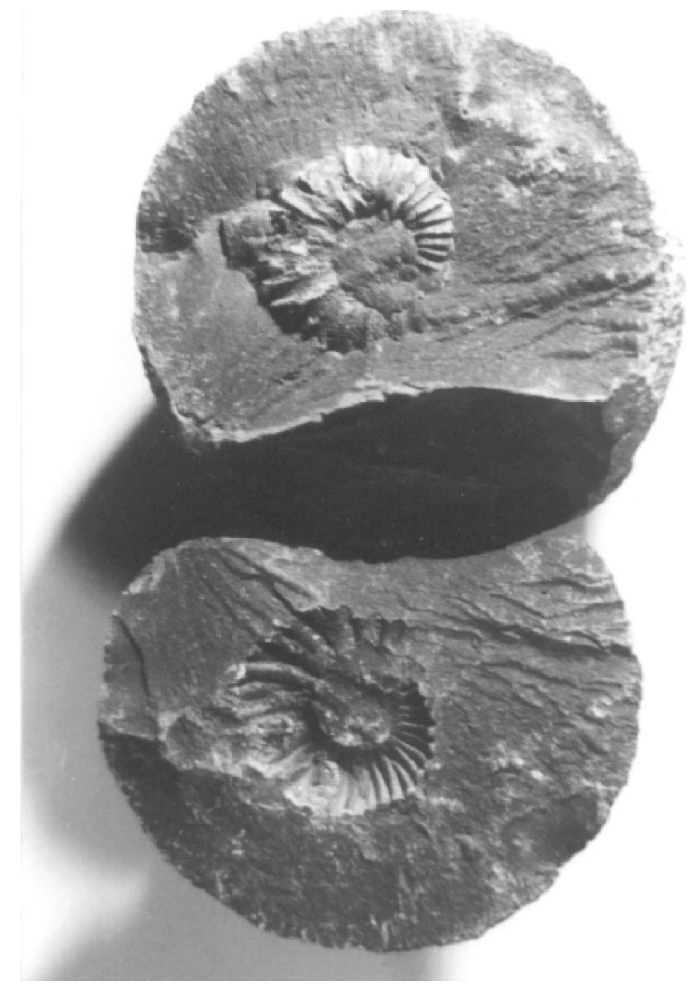

FIG. 4. Analogy between the two halves of a broken fossil and quantum entangled images (see text).

precise prescription for the phase $\phi_{L}$ of the local oscillator (see introduction) in order to observe maximal correlation between symmetrical pixels of the two output images. The optimal value for the phase is that which corresponds to the amplitude fluctuations of the output images in the phasesensitive configuration, when the phase of the symmetrical input images is selected to obtain maximal amplification.

We have also shown that a performance very close to that of the ideal case of the optimal LO phase may be obtained by using a LO with a quadratic wave front (as in Gaussian beams), with the curvature used as optimization parameter.

The connection between quadrature fluctuations and amplitude/phase fluctuations allowed us to conclude also that, while intensity fluctuations are strongly correlated in the twin images, phase fluctuations are strongly anticorrelated in the same amount. An amusing analogy with amplitude and phase fluctuations in entangled twin images is provided by a fossil broken in two pieces (see Fig. 4). We see that the structures of the two pieces have the same "amplitude" = thickness, but opposite "phase" (one is concave and the other convex, one is right handed and the other, left handed).

It is important to underline that while the results for intensity and phase fluctuations hold only in the presence of an input image, the result on EPR entanglement of quadrature components hold also in the absence of any input image, i.e., in the case of the pure parametric down conversion as in [16]. This is important for the applications to quantum teleportation of optical images [3], as a generalization of the Braunstein-Kimble $[18,19]$ scheme for a single-mode field, or to quantum cryptography with images.

The results shown in this paper may be generalized to the 
case of type II phase matching, in which the two entangled images are created in orthogonal polarization. The entanglements is still between symmetric pixels of the two images, but the gain functions have no longer rotational symmetry around the $z$ axis. This case will be treated in details in a subsequent paper.

We observe finally that our results hold also when the OPA is replaced by an optical parametric oscillator below threshold with plane mirrors (see [5] in this connection). As a matter of fact, also in this case, one has an input-output relation of the form (7), and the results are based only on this relation and on the general properties of the functions $\bar{u}$ and $\bar{v}$.

\section{ACKNOWLEDGMENT}

This work was supported by the network QSTRUCT of the TMR program of the EU.

\section{APPENDIX}

In this Appendix, we give the details of the calculation leading to the fluctuation spectra of the photocurrent difference measured on symmetrical regions of the output field, considering first the case of homodyne detection [Eqs. (23)], and then the case of direct intensity measurements (Eq. (38)).

\section{Derivation of Eq. (23)}

Definitions (20) and (21) may be used in order to write the fluctuation spectrum (22) in the form

$$
\begin{aligned}
& V_{\phi_{L}}^{( \pm)}(\Omega)=\int_{R_{1}} d \vec{x} \int_{R_{1}} d \vec{x}^{\prime} \int_{-\infty}^{\infty} d t e^{i \Omega t}\left\langle\delta \hat{Z}_{\phi}(\vec{x}, t) \delta \hat{Z}_{\phi}\left(\vec{x}^{\prime}, 0\right)\right\rangle \\
& \quad+\int_{R_{1}} d \vec{x} \int_{R_{2}} d \vec{x}^{\prime} \int_{-\infty}^{\infty} d t e^{i \Omega t}\left\langle\delta \hat{\mathrm{Z}}_{\phi}(\vec{x}, t) \delta \hat{\mathrm{Z}}_{\phi}\left(\vec{x}^{\prime}, 0\right)\right\rangle \\
& \quad+\left(R_{1} \leftrightarrow R_{2}\right) .
\end{aligned}
$$

The double integrals on the same pixel areas $\left(R_{1}\right.$ or $\left.R_{2}\right)$ give the contribution of intensity self correlations, while the double integrals on different areas describe cross correlations. Substituting Eqs. (16) and (17) into Eq. (18) and putting $a_{L}(\vec{x}, t)=\alpha_{L}(\vec{x})+\delta a_{L}(\vec{x}, t)$, we obtain

$$
\begin{aligned}
Z_{\phi_{L}}(\vec{x}, t)= & \eta\left[a_{4}^{\dagger}(\vec{x}, t) \alpha_{L}(\vec{x})+a_{4}(\vec{x}, t) \alpha_{L}^{*}(\vec{x})\right] \\
& +\eta\left[a_{4}^{\dagger}(\vec{x}, t) \delta a_{L}(\vec{x}, t)+a_{4}(\vec{x}, t) \delta a_{L}^{\dagger}(\vec{x}, t)\right] \\
& +\sqrt{\frac{\eta(1-\eta)}{2}}\left\{\left[a_{4}^{\dagger}(\vec{x}, t)+a_{L}^{\dagger}(\vec{x}, t)\right] a_{N}^{b}(\vec{x}, t)\right. \\
& \left.+\left[a_{4}(\vec{x}, t)+a_{L}(\vec{x}, t)\right] a_{N}^{b \dagger}(\vec{x}, t)\right\} \\
& -\sqrt{\frac{\eta(1-\eta)}{2}}\left\{\left[a_{4}^{\dagger}(\vec{x}, t)-a_{L}^{\dagger}(\vec{x}, t)\right] a_{N}^{c}(\vec{x}, t)\right.
\end{aligned}
$$

$$
\begin{aligned}
& \left.+\left[a_{4}(\vec{x}, t)-a_{L}(\vec{x}, t)\right] a_{N}^{c \dagger}(\vec{x}, t)\right\}+(1-\eta) \\
& \times\left[a_{N}^{b \dagger}(\vec{x}, t) a_{N}^{b}(\vec{x}, t)-a_{N}^{c \dagger}(\vec{x}, t) a_{N}^{c}(\vec{x}, t)\right] .
\end{aligned}
$$

We now use this expression in order to evaluate the corresponding fluctuation correlation function $\left\langle\delta Z_{\phi_{L}}(\vec{x}, t) Z_{\phi_{L}}\left(\vec{x}^{\prime}, t^{\prime}\right)\right\rangle$ appearing on the r.h.s. of Eq. (A1). Noting that most terms containing the annihilation operators $a_{N}^{b, c}(\vec{x}, t)$ vanish, being these applied to the vacuum state, and using the relations $\left\langle a_{N}^{b, c}(\vec{x}, t) a_{N}^{b, c} \dagger^{\dagger}\left(\vec{x}^{\prime}, t^{\prime}\right)\right\rangle$ $=\left\langle\delta a_{L}(\vec{x}, t) \delta a_{L}^{\dagger}\left(\vec{x}^{\prime}, t^{\prime}\right)\right\rangle=\delta\left(\vec{x}-\vec{x}^{\prime}\right) \delta\left(t-t^{\prime}\right)$ we get

$$
\begin{aligned}
\left\langle\delta Z_{\phi_{L}}(\vec{x}, t) \delta Z_{\phi_{L}}\left(\vec{x}^{\prime}, t^{\prime}\right)\right\rangle \\
=\eta^{2}\left\langle\left[\delta a_{4}^{\dagger}(\vec{x}, t) \alpha_{L}(\vec{x})+\delta a_{4}(\vec{x}, t) \alpha_{L}^{*}(\vec{x})\right]\right. \\
\left.\quad \times\left[\delta a_{4}^{\dagger}\left(\vec{x}^{\prime}, t^{\prime}\right) \alpha_{L}\left(\vec{x}^{\prime}\right)+\delta a_{4}\left(\vec{x}^{\prime}, t^{\prime}\right) \alpha_{L}^{*}\left(\vec{x}^{\prime}\right)\right]\right\rangle \\
\quad+\eta\left[\left\langle a_{4}^{\dagger}(\vec{x}, t) a_{4}(\vec{x}, t)\right\rangle+(1-\eta)\left|\alpha_{L}(\vec{x})\right|^{2}\right] \\
\quad \times \delta\left(\vec{x}-\vec{x}^{\prime}\right) \delta\left(t-t^{\prime}\right),
\end{aligned}
$$

where $\delta a_{4}(\vec{x}, t)=a_{4}(\vec{x}, t)-\left\langle a_{4}(\vec{x}, t)\right\rangle=a_{4}(\vec{x}, t)-\alpha_{\text {out }}(\vec{x})$. Using input-output relation (7) written here for the fluctuation operators $\delta a_{4}(\vec{x}, t)$ and $\delta a_{1}(\vec{x}, \Omega)=a_{1}(\vec{x}, \Omega)-\alpha_{i n}(\vec{x})$

$$
\delta a_{4}(\vec{x}, \Omega)=\bar{u}(\vec{x}, \Omega) \delta a_{1}(-\vec{x}, \Omega)+\bar{v}(\vec{x}, \Omega) \delta a_{1}^{\dagger}(\vec{x},-\Omega)
$$

the Fourier transformation of Eq. (A4) yields

$$
\begin{aligned}
\int_{-\infty}^{\infty} d t & e^{i \Omega t}\left\langle\delta \hat{z}_{\phi}(\vec{x}, t) \delta \hat{z}_{\phi}\left(\vec{x}^{\prime}, 0\right)\right\rangle \\
= & \eta^{2} \delta\left(\vec{x}-\vec{x}^{\prime}\right)\left[|\vec{u}(\vec{x}, \Omega)|^{2}+|\bar{v}(\vec{x},-\Omega)|^{2}\right]\left|\alpha_{L}(\vec{x})\right|^{2} \\
& +\eta^{2} \delta\left(\vec{x}+\vec{x}^{\prime}\right)\left[\bar{u}(\vec{x}, \Omega) \bar{v}(\vec{x},-\Omega) \alpha_{L}^{*}(-\vec{x}) \alpha_{L}^{*}(\vec{x})\right. \\
& + \text { c.c. }]+\eta(1-\eta)\left|\alpha_{L}(\vec{x})\right|^{2} \delta\left(\vec{x}-\vec{x}^{\prime}\right)+\eta \\
& \times\left\langle a_{4}^{\dagger}(\vec{x}, t) a_{4}(\vec{x}, t)\right\rangle \delta\left(\vec{x}-\vec{x}^{\prime}\right) .
\end{aligned}
$$

Since we have assumed the LO field is much more intense than the output field, the last term of this expression can be neglected. When substituted into the r.h.s. of Eq. (A1), the terms proportional to $\delta\left(\vec{x}-\vec{x}^{\prime}\right)$ give the self-correlations contribution to the spectrum, while the term proportional to $\delta\left(\vec{x}+\vec{x}^{\prime}\right)$ give the cross-correlation contribution. The final result may be written as

$$
V_{\phi_{L}}^{( \pm)}(\Omega)=\eta(1-\eta) \int_{R_{1}+R_{2}} d \vec{x}\left|\alpha_{L}(\vec{x})\right|^{2}
$$




$$
\begin{aligned}
& +\eta^{2} \int_{R_{1}+R_{2}} d \vec{x}\left[\left(|\vec{u}(\vec{x}, \Omega)|^{2}+|\bar{v}(\vec{x}, \Omega)|^{2}\right)\left|\alpha_{L}(\vec{x})\right|^{2}\right] \\
& \pm \eta^{2} \int_{R_{1}+R_{2}} d \vec{x}\left[\bar{u}(\vec{x}, \Omega) \bar{v}(\vec{x},-\Omega) \alpha_{L}^{*}(\vec{x}) \alpha_{L}^{*}(-\vec{x})+\text { c.c. }\right]
\end{aligned}
$$

The first term on the r.h.s is the shot noise level determined by mean intensity of the LO field, while the remaining terms can easily be identified with the last term on the r.h.s of [Eq. (23b)]. Finally, we note that the substitution $\phi_{L} \rightarrow \phi_{L}+\pi / 2$ with $\phi_{L}=\arg \left(\alpha_{L}\right)$ leaves the expression on the r.h.s. unchanged except for the sign of the last term and Eq. (23a) follows.

\section{Derivation of Eq. (38)}

We now outline the derivation of Eq. (38) that gives the fluctuation spectrum of the photocurrent difference $i_{-}(t)$. Using the relation (13), and recalling that $a_{N}(\vec{x}, t)$ is an operator that acts on the vacuum field, so that $\left\langle a_{N}(\vec{x}, t) a_{N}^{\dagger}\left(\vec{x}^{\prime}, t^{\prime}\right)\right\rangle=\delta\left(\vec{x}-\vec{x}^{\prime}\right) \delta\left(t-t^{\prime}\right)$, we can write

$$
\begin{aligned}
\int_{-\infty}^{\infty} d t e^{i \Omega t}\left\langle\delta i_{-}(t) \delta i_{-}(0)\right\rangle \\
\quad=\eta^{2} \int_{-\infty}^{\infty} d t e^{i \Omega t}\left\langle\delta i_{4}^{(-)}(t) \delta i_{4}^{(-)}(0)\right\rangle+(1-\eta)\left\langle i_{+}\right\rangle,
\end{aligned}
$$

where

$$
\begin{gathered}
\delta i_{4}^{(-)}(t)=i_{4}^{(-)}(t)-\left\langle i_{4}^{(-)}(t)\right\rangle, \\
i_{4}^{(-)}(t)=\left.i_{-}(t)\right|_{\eta=1}=\int_{R_{1}} d \vec{x} a_{4}^{\dagger}(\vec{x}, t) a_{4}(\vec{x}, t) \\
\\
-\int_{R_{2}} d \vec{x} a_{4}^{\dagger}(\vec{x}, t) a_{4}(\vec{x}, t),
\end{gathered}
$$

denotes the photocurrent difference fluctuation operator for $\eta=1$. Substituting the expression $a_{4}(\vec{x}, t)=\left\langle a_{4}(\vec{x}, t)\right\rangle$ $+\delta a_{4}(\vec{x}, t)=\alpha_{\text {out }}(\vec{x})+\delta a_{4}(\vec{x}, t)$ into definition (A10), we find

$$
\delta i_{4}^{(-)}(t)=\delta Z_{\text {out }}^{(-)}(t)+\delta i_{v}^{(-)}(t)
$$

where

$$
Z_{\text {out }}^{(-)}(t)=Z_{\text {out }}^{(1)}(t)-Z_{\text {out }}^{(2)}(t)
$$

$$
Z_{\text {out }}^{(i)}(t)=\int_{R_{i}} d \vec{x}\left[a_{4}^{\dagger}(\vec{x}, t) \alpha_{\text {out }}(\vec{x})+a_{4}(\vec{x}, t) \alpha_{o u t}^{*}(\vec{x})\right]
$$

is the difference between the quadrature amplitude fluctuation operators, while $\delta i_{v}^{(-)}(t)=i_{v}^{(-)}(t)-\left\langle i_{v}^{(-)}(t)\right\rangle$ with

$$
i_{v}^{(-)}(t)=\int_{R_{1}} d \vec{x} \delta a_{4}^{\dagger}(\vec{x}, t) \delta a_{4}(\vec{x}, t)-\int_{R_{2}} d \vec{x} \delta a_{4}^{\dagger}(\vec{x}, t) \delta a_{4}(\vec{x}, t)
$$

gives the pure noise contribution to the photocurrent fluctuation spectrum arising from spontaneous down conversion (this term is the only one present if the input is in the vacuum state). Since any product of an odd number of fluctuation operators has zero expectation value, there is no correlation between $\delta Z_{\text {out }}^{(-)}(t)$ and $i_{v}^{(-)}(t)$, and we may write

$$
\begin{gathered}
\int_{-\infty}^{\infty} d t e^{i \Omega t}\left\langle\delta i_{4}^{(-)}(t) \delta i_{4}^{(-)}(0)\right\rangle \\
=\int_{-\infty}^{\infty} d t e^{i \Omega t}\left\langle\delta Z_{\text {out }}^{(-)}(t) \delta Z_{\text {out }}^{(-)}(0)\right\rangle \\
+\int_{-\infty}^{\infty} d t e^{i \Omega t}\left\langle\delta i_{v}^{(-)}(t) \delta i_{v}^{(-)}(0)\right\rangle .
\end{gathered}
$$

The quadrature fluctuations spectrum has been already calculated in the first part of the Appendix; its contribution to Eq. (38) may be identified by looking at Eqs. (40), and (41), which represent the limit of the intensity difference noise when spontaneously down-converted photons may be neglected.

The spectrum of $i_{v}^{(-)}(t)$ is more cumbersome to evaluate since it involves the calculation of the fourth-order field correlation function $\left\langle\delta a_{4}^{\dagger}(\vec{x}, t) \delta a_{4}(\vec{x}, t) \delta a_{4}^{\dagger}\left(\vec{x}^{\prime}, t^{\prime}\right) \delta a_{4}\left(\vec{x}^{\prime}, t^{\prime}\right)\right\rangle$. A direct substitution of input-output relation (A5) leads to the last integral on the r.h.s. of Eq. (38) multiplied by $\delta(\vec{x}$ $=0)$. This divergence finds its explanation in the nonphysical assumption that the pump field is a plane wave, thus able to provide an infinite number of spontaneously downconverted photon pairs. The finite transverse dimensions of the system can be taken into account by introducing a pupil at the output face of the crystal (see [10] for more details). If the pupil area $S_{p}$ is sufficiently large, the corresponding diffraction spot area $S_{R}=(\lambda f)^{2} / S_{p}$ is much smaller than the coherence area of the amplifier $x_{0}^{2}$ and it can be shown that in this limit, $\delta(0)$ is symply replaced by the factor $1 / S_{R}$ (see [16] for a full calculation).
[1] T.B. Pittman, Y.H. Shih, D.V. Strekalov, and A.V. Sergienko, Phys. Rev. A 52, R3429 (1995); T.B. Pittman, D.V. Strekalov, D.N. Klyshko, M.H. Rubin, A.V. Sergienko, and Y.H. Shih, ibid. 53, 2804 (1996); B.M. Jost, A.V. Sergienko, A.F. Abouraddy, B.E.A. Saleh, and M.C. Teich, Opt. Express 3, 81 (1998); B.E.A. Saleh, A. Joobeur, and M. Teich, Phys. Rev. A
57, 3991 (1998); B.E.A. Saleh, A. Abouraddy, A.V. Sergienko, and M. Teich, ibid. 62, 043816 (2000).

[2] C.H. Monken, P.H. Souto Ribeiro, and S. Padua, Phys. Rev. A 57, 3123 (1998); E.J.S. Fonseca, C.H. Monken, S. Padua, and G.A. Barbosa, ibid. 59, 1608 (1999); P.H. Souto Ribeiro, S. Padua, and C.H. Monken, ibid. 60, 5074 (1999). 
[3] I. V. Sokolov, M. I. Kolobov, A. Gatti, and L. A. Lugiato, Opt. Commun., (to be published).

[4] C. Fabre, J.B. Fouet, and A. Maitre, Opt. Lett. 25, 76 (2000).

[5] A. Gatti, E. Brambilla, L.A. Lugiato, and M.I. Kolobov, Phys. Rev. Lett. 83, 1763 (1999).

[6] A. Gatti, E. Brambilla, L. A. Lugiato, and M. H. Kolobov, J. Opt. B: Quantum Semiclass. Opt. (to be published).

[7] A. Einstein, B. Podolsky, and N. Rosen, Phys. Rev. 47, 777 (1935)

[8] M.D. Reid, Phys. Rev. A 40, 913 (1989); Z.Y. Ou, S.F. Pereira, and H.J. Kimble, Appl. Phys. B: Photophys. Laser Chem. 55, 265 (1992).

[9] A. Gatti, L.A. Lugiato, K.I. Petsas, and I. Marzoli, Europhys. Lett. 46, 461 (1991); Opt. Commun. (to be published).

[10] I.V. Sokolov, M.I. Kolobov, and L.A. Lugiato, Phys. Rev. A 60, 2420 (1999).
[11] M.I. Kolobov, and I.V. Sokolov, Sov. Phys. JETP 69, 1097 (1989); Phys. Lett. A 140, 101 (1989).

[12] M.I. Kolobov and L.A. Lugiato, Phys. Rev. A 52, 4930 (1995).

[13] J.A. Levenson, I. Abram, T. Rivera, P. Fayolle, J.C. Garreau, and Ph. Grangier, Phys. Rev. Lett. 70, 267 (1993).

[14] Sang-Kyung Choi, M Vasilyev, and P. Kumar, Phys. Rev. Lett. 83, 1938 (1999).

[15] C.M. Caves, Phys. Rev. D 26, 1817 (1982)

[16] E. Brambilla, A. Gatti, and L.A. Lugiato, Eur. Phys. J. D 15, 127 (2001).

[17] See, e.g., F. Devaud and F. Lantz, J. Opt. Soc. Am. B 12, 2245 (1995).

[18] S.L. Braunstein and H.J. Kimble, Phys. Rev. Lett. 80, 869 (1998); A. Furusawa, J.L. Sorensen, S.L. Braunstein, C.A. Fuchs, H.J. Kimble, and E.S. Polzik, Science 282, 706 (1998).

[19] L. Vaidman, Phys. Rev. A 49, 1473 (1994). 